\section{le Scale}

\begin{tabular}{|c|c|c|c|}
\hline & $\begin{array}{l}\text { 1) case definition } \\
\text { validation }\end{array}$ & $\begin{array}{l}\text { 2) Representative- } \\
\text { ness }\end{array}$ & $\begin{array}{l}\text { 3) selection of } \\
\text { controls }\end{array}$ \\
\hline Booker 2016 & & * & * \\
\hline Burton 2012 & & * & * \\
\hline Davies 2011 & * & * & * \\
\hline Dregan 2015 anti-infla & am drugs & * & * \\
\hline Dunn 2005 Lithium & * & * & * \\
\hline Dunn 2005 Infectious I & ।* & * & * \\
\hline Imfeld 2012 & * & * & * \\
\hline Imfeld 2013 Seizures & * & * & * \\
\hline Imfeld 2013 Epidemiol & * & * & * \\
\hline Imfeld 2015 & * & * & * \\
\hline Imfeld 2016 & * & * & * \\
\hline Jick 2000 & * & & $*$ \\
\hline Ramakers 2007 & & * & $*$ \\
\hline Seshadri 2001 & * & * & * \\
\hline Wagner 2012 & & * & * \\
\hline
\end{tabular}

Cohort studies Selection
1)

ss of the exposed Selection of non- ascertainment of outcome not cohort exposed cohort exposure

4) definition of
controls
$*$
$*$
$*$
$*$
$*$
$*$
$*$
$*$
$*$
$*$
$*$
$*$
$*$

Comparability

1) Comparability

of cases and

controls

**

**

$* *$
$* *$

$*$

$* *$
$* *$

**

**

**

$* *$
$* *$

\section{Comparability}

Comparability of

cohorts on basis

of design

analysis

Buntinx 1996

Dregan 2015 inflamme *

Kessing 2008

Koehler 2015
$*$
$*$
$*$
Exposure

2)

1) Ascertainment for cases and

of Exposure

controls response rate

3) Non-

*

$*$
$*$

Asses

Assessment of Follow up long Adequacy of enough $>10$ years follow up
RoBANS

Selection

of Confound Measure outcome Incmplete Selective participan ing ment of assessme outcome outcome ts Variables exposure nts data reporting 8 low risk low risk low risk low risk low risk low risk 8 low risk low risk low risk low risk low risk low risk 8 low risk low risk low risk low risk low risk low risk 8 low risk low risk low risk low risk low risk low risk 8 low risk unclear ris low risk low risk low risk low risk 9 low risk low risk low risk low risk low risk low risk 9 low risk low risk low risk low risk low risk low risk 9 low risk low risk low risk low risk low risk low risk 9 low risk low risk low risk low risk low risk low risk low risk low risk low risk low risk low risk low risk glow risk low risk lowisk low risk low risk low risk 9 low risk low risk low risk low risk low risk low risk 8 unclear low risk low risk low risk low risk low risk 8 low risk low risk low risk low risk low risk low risk 8 low risk low risk low risk low risk low risk low risk 8 low risk low risk low risk low risk low risk low risk
8 low risk low risk low risk low risk low risk low risk 7 low risk low risk low risk low risk low risk low risk 9 low risk low risk low risk low risk low risk low risk 8 low risk unclear ris low risk low risk low risk low risk 